\title{
Switching Control for Multi-Scroll Chaos Generation: An Overview
}

\author{
Jinhu L $\ddot{u}^{1} \quad$ Xinghuo $\mathrm{Yu}^{2} \quad$ Guanrong $\mathrm{Chen}^{3}$ \\ ${ }^{1}$ Institute of Systems Science, Academy of Mathematics and System Sciences \\ Chinese Academy of Sciences, Beijing 100080, China \\ ${ }^{2}$ Faculty of Engineering, RMIT University, Melbourne, Australia \\ ${ }^{3}$ Department of Electronic Engineering, City University of Hong Kong, Hong Kong \\ lvjinhu@mail.amss.ac.cn; x.yu@rmit.edu.au; gchen@ee.cityu.edu.hk
}

\begin{abstract}
This paper reviews and meanwhile introduces several new switching piecewise-linear controllers, which can generate multi-scroll chaotic attractors from some simple two-dimensional (2D) or three-dimensional (3D) linear autonomous systems. The mechanism for generating multi-scroll chaotic attractors via switching control is discussed.
\end{abstract}

\section{Introduction}

Exploiting chaotic dynamics in high-tech and industrial engineering applications has attracted more and more interest. In particular, attention has been focused on effectively generating chaos using some simple devices such as nonlinear circuits and switching controllers [1]-[12].

It is well known that piecewise-linear functions can easily create various chaotic attractors, typically the $n$-scroll attractors of Chua's circuits [2]-[3]. Nowadays, generating multi-scroll chaotic attractors is no longer a very difficult task, and there have been some successful results reported in the literature [1]-[12]. These methods can be classified as: (1) adding breakpoints [1]-[6]; (2) switching manifold method [7]-[8]; (3) using hysteresis series switching [9]-[11]; (4) using sine functions [12]. In the following, we review and meanwhile introduce some approaches for generating multi-scroll chaotic attractors from simple linear autonomous systems via switching control.

This paper is organized as follows: Chua's circuit and the generalized Chua's circuit are first reviewed in Section 2. Section 3 introduces the recently proposed family of scroll grid chaotic attractors. A new switching control approach for generating chaotic attractors with multiple merged basins of attraction is then introduced in Section 4. Section 5 presents a sys- tematic method for generating multi-scroll chaotic attractors via hysteresis switching. Conclusions are finally given in Section 6.

\section{The $n$-scroll Attractors of Chua's Circuits}

This section reviews the familiar Chua's circuit and the so-called generalized Chua's circuit.

Chua's circuit is a simple autonomous system, which can exhibit a double-scroll chaotic attractor [1]. The state equations of the circuit are described by

$$
\left\{\begin{array}{l}
\dot{x}=\alpha(-x+y-f(x)) \\
\dot{y}=x-y+z \\
\dot{z}=-\beta y
\end{array}\right.
$$

where

$$
f(x)=m_{0} x+\frac{1}{2}\left(m_{1}-m_{0}\right)(|x+1|-|x-1|) .
$$

When $\alpha=10, \beta=14.87, m_{0}=-0.68, m_{1}=-1.27$, system (1) displays a double-scroll chaotic attractor.

Suykens et al [2] proposed a generalized Chua's circuit, described by

$$
\left\{\begin{array}{l}
\dot{x}=\alpha(y-h(x)) \\
\dot{y}=x-y+z \\
\dot{z}=-\beta y
\end{array}\right.
$$

where the nonlinear function $h(\cdot)$ is given by the following switching piecewise-linear characteristic function with multiple breakpoints:

$$
\begin{aligned}
h(x)= & m_{2 q-1} x+\sum_{i=1}^{2 q-1}\left(m_{i-1}-m_{i}\right) \\
& \times\left(\left|x+c_{i}\right|-\left|x-c_{i}\right|\right)
\end{aligned}
$$

in which $q$ is a positive integer, and $\alpha, \beta, q, m_{k}$ and $c_{l}$ are constant coefficients. System (2) becomes system (1) if $q=1$. System (2) can exhibit an even or 
$\alpha=9, \beta=14.286, q=2, m_{0}=\frac{9}{70}, m_{1}=-\frac{3}{7}$, $m_{2}=\frac{1}{2}, m_{3}=-\frac{2.4}{7}, c_{1}=1, c_{2}=2.15, c_{3}=4$,

system (2) exhibits a 3-scroll chaotic attractor as shown in Figure 1.

\section{Remarks 1:}

(1) System (2)-(3) is continuous and can exhibit an $n$-scroll chaotic attractor for any $n \in N$.

(2) Suykens and Vandewalle [3] introduced some breakpoints in the piecewise-linear characteristic of the nonlinear resistor of Chua's circuit, to obtain a maximum of six double-scrolls attractors. Aziz-Alaoui [4] produced a 10-spiral attractor from Chua's circuit from a similar approach. Recently, Zhong et al [5] proposed a systematic approach for generating $n$-scroll attractors based on Chua's circuit, which can generate e.g. a 10-scroll attractor on an oscilloscope as shown in Figure 2.

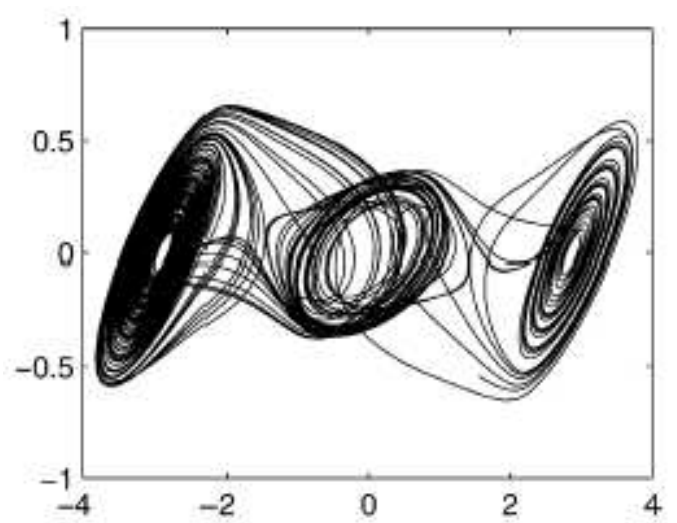

Figure 1. The 3-scroll chaotic attractor.

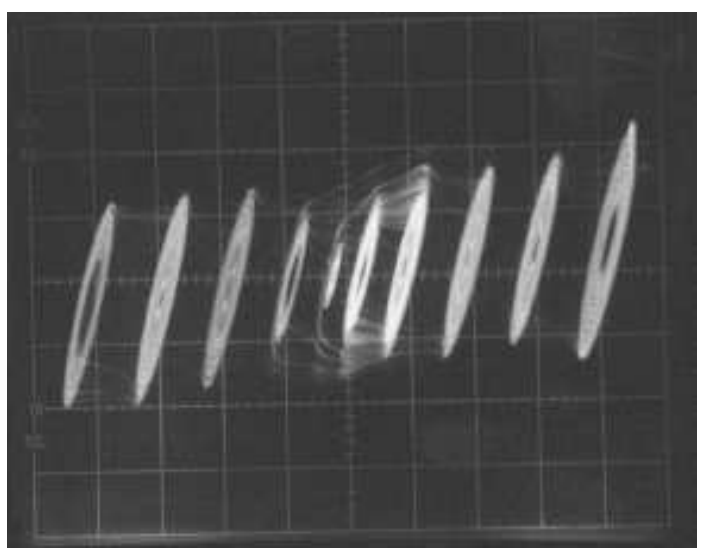

Figure 2. The 10-scroll chaotic attractor on an oscilloscope.
Recently, Yalcin et al [6] introduced a new family of scroll grid attractors, which are classified into 1D, $2 \mathrm{D}$, and 3D-grid scroll chaotic attractors, depending on the location of the equilibrium points in the state space. The state equation of this family of systems is described by

$$
\dot{X}=A X+B \sigma(C X)
$$

where

$$
\begin{gathered}
A=\left(\begin{array}{ccc}
0 & 1 & 0 \\
0 & 0 & 1 \\
-a & -a & -a
\end{array}\right), B=\left(\begin{array}{ccc}
b_{y} & 0 & 0 \\
0 & b_{z} & 0 \\
0 & 0 & a
\end{array}\right), \\
C=\left(\begin{array}{ccc}
0 & 1 & 0 \\
0 & 0 & 1 \\
1 & 0 & 0
\end{array}\right), X=\left(\begin{array}{l}
x \\
y \\
z
\end{array}\right) .
\end{gathered}
$$

There are three different cases:

- 1D-scroll grid attractors (n-scroll attractors):

$$
\left\{\begin{array}{c}
b_{y}=b_{z}=0, \\
\sigma(\cdot)=\left(\begin{array}{c}
0 \\
0 \\
f_{1}(\cdot)
\end{array}\right),
\end{array}\right.
$$

where

$$
f_{1}(x)=\sum_{i=1}^{M_{x}} g_{\frac{-2 i+1}{2}}(x)+\sum_{i=1}^{N_{x}} g_{\frac{2 i-1}{2}}(x),
$$

and

$$
g_{\theta}(\zeta)=\left\{\begin{array}{c}
1, \quad \zeta \geq \theta \quad \theta>0 \\
0, \quad \zeta<\theta \quad \theta>0 \\
0, \quad \zeta \geq \theta \quad \theta<0 \\
-1, \quad \zeta<\theta \quad \theta<0
\end{array}\right.
$$

which belongs to the sector $[0,2]$.

- 2D-scroll grid attractors:

$$
\left\{\begin{array}{c}
b_{y}=-1, b_{z}=0 \\
\sigma(\cdot)=\left(\begin{array}{c}
f_{1}(\cdot) \\
0 \\
f_{2}(\cdot)
\end{array}\right)
\end{array}\right.
$$

where

$$
f_{2}(x)=\sum_{i=1}^{m-1} \beta g_{p_{i}}(x)
$$

and

$$
\begin{aligned}
& p_{i}=M_{y}+0.5+(i-1)\left(M_{y}+N_{y}+1\right), \\
& \beta=M_{y}+N_{y}+1,
\end{aligned}
$$

which belongs to the sector $\left[0, \frac{M_{y}+N_{y}+1}{M_{y}+0.5}\right]$. 
- 3D-scroll grid attractors:

$$
\left\{\begin{array}{l}
b_{y}=-1, b_{z}=-1, \\
\sigma(\cdot)=\left(\begin{array}{l}
f_{1}(\cdot) \\
f_{1}(\cdot) \\
f_{3}(\cdot)
\end{array}\right)
\end{array}\right.
$$

where

$$
f_{3}(x)=\sum_{i=1}^{k-1} \gamma g_{n_{l}}(x)
$$

and

$n_{l}=\rho+0.5+(l-1)(\rho+\zeta+1), \gamma=\rho+\zeta+1$,

with

$$
\begin{aligned}
& \rho=\left|\min _{i, j}\left\{u_{i}^{e q, y}+u_{j}^{e q, z}\right\}\right|, \\
& \zeta=\left|\max _{i, j}\left\{u_{i}^{e q, y}+u_{j}^{e q, z}\right\}\right|,
\end{aligned}
$$

and $u_{i}^{e q, y}$ and $u_{j}^{e q, z}$ are the vectors for the $y$ and $z$ variables related to the equilibrium points, which belongs to the sector $\left[0, \frac{\zeta+\rho+1}{\rho+0.5}\right]$.

Figure 3 (a) shows a 10-scroll chaotic attractor, where $M_{x}=0, N_{x}=1, a=0.8, M=4, N=$ $5, X_{0}=\left[\begin{array}{lll}0.6721 & 0.8381 & 0.0196\end{array}\right]$. Figure 3 (b) displays a $2 \times 3$-grid scroll attractor, where $M_{y}=$ $0, N_{y}=2, m=2$. Figure 3 (c) shows a $4 \times 3 \times 2$ grid scroll attractor, where $M_{y}=1, N_{y}=1, M_{x}=$ $0, N_{x}=1, k=4$.

\section{Remarks 2:}

(1) It is possible to generate chaotic attractors whose scrolls are located in any position with any orientation.

(2) System (4) is not continuous. However, the generalized Chua's circuit (2) is continuous.

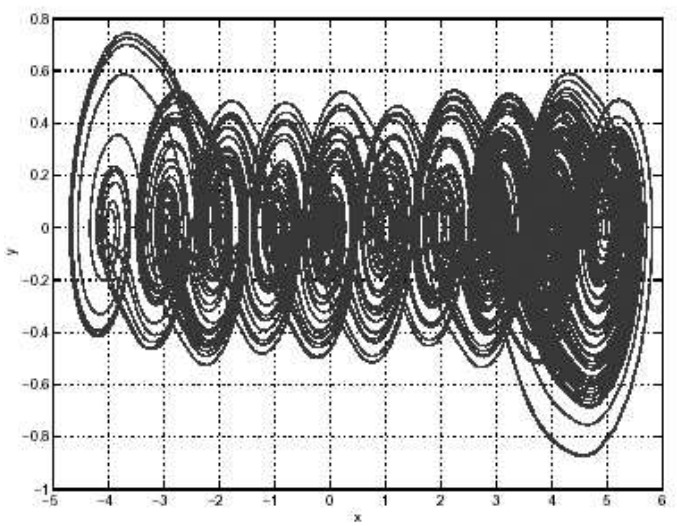

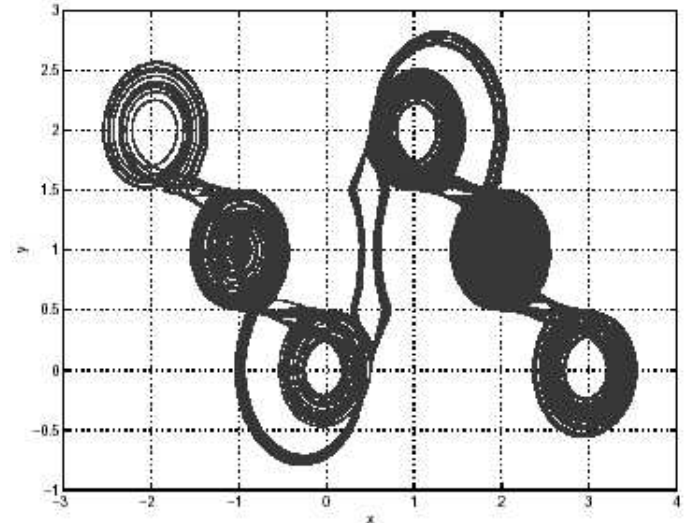

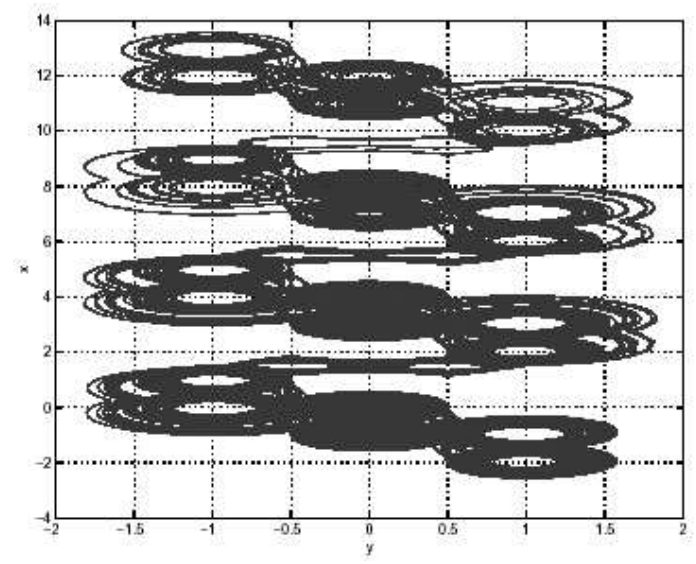

Figure 3. (a) 10 -scroll attractor, (b) $2 \times 3$-grid scroll attractor, (c) $4 \times 3 \times 2$-grid scroll attractor.

\section{Multiple Merged Basins of Attraction Chaotic Attractors}

This section introduces a new switching control approach for generating $n$-scroll chaotic attractors from a 3D linear autonomous system.

Recently, Lü et al [7, 8] presented a systematic switching piecewise-linear control method for creating strange attractors with multiple merged basins of attraction, by the following 3D linear controlled system:

$$
\dot{X}=A X+U,
$$

where $X=(x, y, z)^{T}$ and

$$
A=\left(\begin{array}{ccc}
a & b & 0 \\
-b & a & 0 \\
0 & 0 & c
\end{array}\right)
$$

with a switching piecewise-linear controller

$U=f_{1}(X)= \begin{cases}k\left(\begin{array}{c}-x \\ -y \\ d\end{array}\right), & \text { if } z+\sqrt{x^{2}+y^{2}}>k, \\ 0, & \text { otherwise }\end{cases}$ 
where $a, b, c, d, k$ are real parameters. Under the controller $U=f_{1}(X)$, system (12) can create strange attractors within a wide range of parameter values.

To generate two chaotic attractors simultaneously from system (12), we use the controller $U$ as follows [8]:

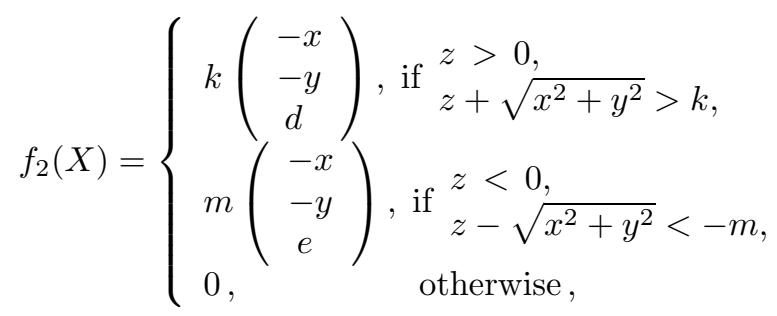

where $a, b, c, d, e, k, m$ are real parameters. Under this controller $U=f_{2}(X)$, system (12) can simultaneously generate two chaotic attractors - an upperattractor and a lower-attractor. Note that $z=0$ is the invariant manifold of system (12) with the controller $U=f_{2}(X)$. Figure 4 displays an upper and a lower chaotic attractors, where $a=3, b=20$, $c=-20, d=10, e=-10, k=4$, and $m=4$.

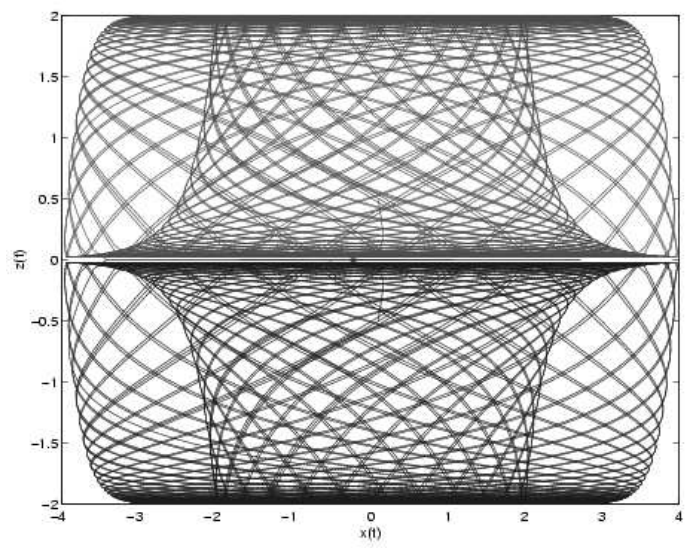

Figure 4. The upper and lower chaotic attractors.

\section{Remarks 3:}

(1) Similarly, we can easily generate $n$ different attractors simultaneously with different initial values in the switching system (12) by two fundamental transforms: parallel displacement and rotation. Since the chaotic attractor is bounded by a finite sphere, we can partition the whole space into $n$ disjoint subspaces, and then duplicate the original attractor - the upper attractor or the lower-attractor - into every subspace.

(2) The above $n$ attractors are independent of one another. Moreover, there is no system orbit that connects all attractors together.
To connect together the orbits of the upper- and lower-attractors, so as to obtain a single chaotic attractor, we can design a controller to force the orbit of the upper-attractor to go through the invariant manifold $z=0$ from above and then enter into the subspace $\{(x, y, z) \mid z<0\}$; at the same time, this controller should force the orbit of the lower-attractor to go through the invariant manifold $z=0$ from below and then return to the subspace $\{(x, y, z) \mid z>0\}$. Based on this idea, we use $\delta(0,0,-\operatorname{sign}(z))^{\top}$ to substitute for 0 in the controller $U=f_{2}(X)$, therefore obtaining the following new controller:

$f_{3}(X)=\left\{\begin{array}{l}k\left(\begin{array}{c}-x \\ -y \\ d\end{array}\right), \text { if } \begin{array}{l}z>0, \\ z+\sqrt{x^{2}+y^{2}}>k,\end{array} \\ m\left(\begin{array}{c}-x \\ -y \\ e\end{array}\right), \text { if } \begin{array}{l}z<0 \\ z-\sqrt{x^{2}+y^{2}}<-m,\end{array} \\ \delta\left(\begin{array}{c}0 \\ 0 \\ -\operatorname{sign}(z)\end{array}\right), \text { otherwise }\end{array}\right.$

where $a, b, c, d, e, k, m, \delta$ are all real parameters.

Figure 5 shows that the chaotic attractor has two merged basins of attraction: the upper basin of attraction and the lower basin of attraction.

Note that the controller $U=f_{3}(X)$ has three switching planes: $S_{1}, S_{2}$, and $z=0$, in which the two switching planes $S_{1}$ and $S_{2}$ are responsible for the generation of two chaotic attractors, the upper chaotic attractor and the lower chaotic attractor; while the switching plane $z=0$ is responsible for the connection of these two chaotic attractors.

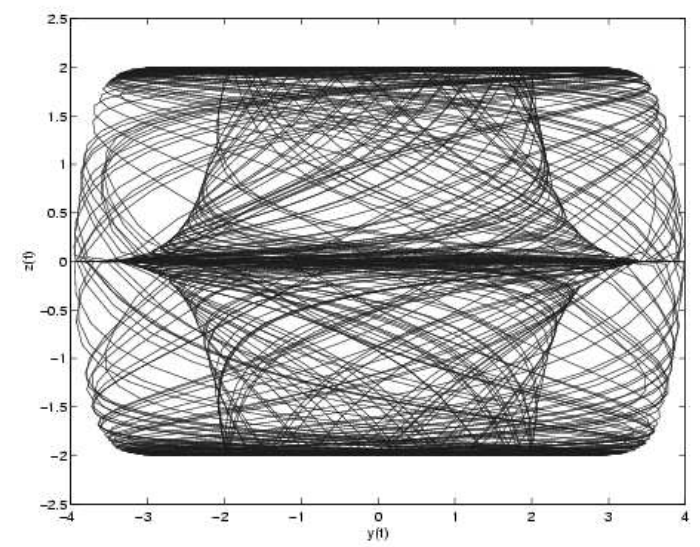

Figure 5. The chaotic attractor with two merged basins of attraction.

To generate a chaotic attractor with three merged basins of attraction, we use $\delta(0,0,-\operatorname{sign}(z-h)-$ $c h / \delta)^{\top}$ to substitute for 0 in the controller, resulting 
in the following new controller:

$$
\begin{aligned}
& f_{4}(X)=
\end{aligned}
$$

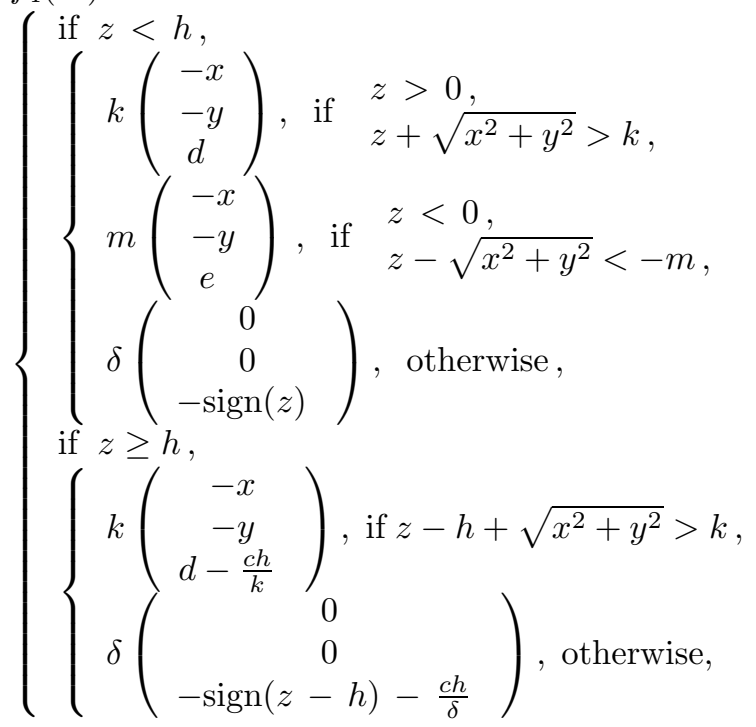

where $a, b, c, d, e, k, m, h, \delta$ are all real parameters.

Figure 6 displays that the chaotic attractor has three merged basins of attraction: two upper basins of attraction and one lower basin of attraction.

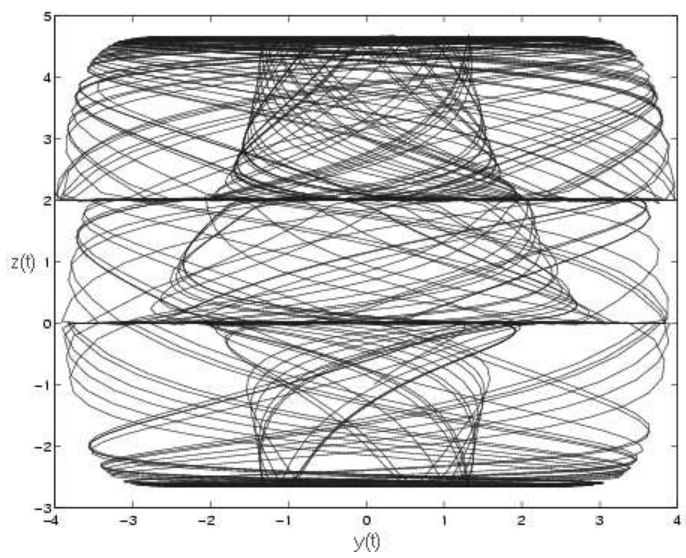

Figure 6. The chaotic attractor with three merged basins of attraction.

Similarly, we can create chaotic attractors with $n$ merged basins of attraction. The formalized design approach is as follows:

(1) Partition the whole space into $n$ subspaces. For convenience, one may partition the space along the $z$-axis.

(2) Duplicate the original attractors, the upperattractor and the lower-attractor, to every subspaces.

(3) Use the switching controller to connect all the $n$ independent attractors, so as to form a single chaotic attractor with multiple merged basins of attraction, as depicted by Figure 7 .

Note that the switching controller can be chosen as $\delta \operatorname{sign}\left(z-h_{i}\right)$, where the height $h_{i}$ (between two neighboring subspaces) should be smaller than the height of a single chaotic attractor.

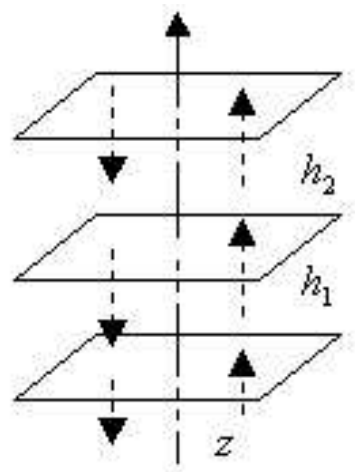

Figure 7. The illustrative sketch for the connection of orbit.

\section{Hysteresis Chaotic Attractors}

This section presents several new hysteresis switching multi-dimensional scroll chaotic attractors [9][11]. We consider two cases: the system to be controlled is a two-dimensional linear autonomous system, and is a three-dimensional linear autonomous system.

\subsection{Two-Dimensional Case}

This subsection introduces a new systematic method for generating multi-scroll chaotic attractors from a two-dimensional linear autonomous system via switching of hysteresis series [10]. It includes 1D $n$-scroll and 2D $n \times m$-grid scroll chaotic attractors.

The hysteresis switching chaotic system, which can be regarded as a linear system with a hysteresis feedback nonlinearity, is described by

$$
\dot{X}=A X+B \theta(C X),
$$

where $X=(x, y)^{T}$ is the state vector, and

$$
\begin{aligned}
& A=\left(\begin{array}{cc}
0 & 1 \\
-a & b
\end{array}\right), \quad B=\left(\begin{array}{cc}
-1 & 0 \\
-b & a
\end{array}\right), \\
& C=\left(\begin{array}{cc}
0 & 1 \\
1 & 0
\end{array}\right) .
\end{aligned}
$$

There are three different cases as follows: 
- 1D horizontal $n$-scroll chaotic attractor:

$$
\theta(\cdot)=\left(\begin{array}{c}
0 \\
h\left(x, p_{1}, q_{1}\right)
\end{array}\right)
$$

where

$$
h\left(x, p_{1}, q_{1}\right)=\sum_{i=1}^{p_{1}} h_{-i}(x)+\sum_{i=1}^{q_{1}} h_{i}(x),
$$

is called a hysteresis series, where $p_{1}$ and $q_{1}$ are positive integers, and

$$
h_{i}(x)= \begin{cases}0 & \text { for } \quad x<i \\ 1 & \text { for } \quad x>i-1\end{cases}
$$

where $h_{i}(x)$ is switched from 1 to 0 if $x$ hits the threshold $i-1$ from above and is switched from 0 to 1 if $x$ hits $i$ from below, and

$$
h_{-i}(x)= \begin{cases}0 & \text { for } \quad x>-i \\ -1 & \text { for } \quad x<-i+1\end{cases}
$$

where $h_{-i}(x)$ is switched from 0 to -1 if $x$ hits the threshold $-i$ from above and is switched from -1 to 0 if $x$ hits $-i+1$ from below. Here, the equilibria are located in the $x$-axis.

- 1D vertical $n$-scroll chaotic attractor:

$$
\theta(\cdot)=\left(\begin{array}{c}
h\left(y, p_{2}, q_{2}\right) \\
0
\end{array}\right)
$$

where $h\left(x, p_{2}, q_{2}\right)$ is similarly defined by (14), whose equilibria are located in the $y$-axis.

- 2D $n \times m$-grid scroll chaotic attractor:

$$
\theta(\cdot)=\left(\begin{array}{c}
h\left(y, p_{2}, q_{2}\right) \\
h\left(x, p_{1}, q_{1}\right)
\end{array}\right)
$$

where $h\left(x, p_{1}, q_{1}\right), h\left(x, p_{2}, q_{2}\right)$ are similarly defined by (14), whose equilibria are integer points in state space.

Figure 8 shows the hysteresis multi-scroll chaotic attractors generated by system (13): (a) a horizontal 7-scroll attractor, where $a=1, b=0.125$, $p=q=3$; (b) a vertical 7 -scroll attractor, where $a=1, b=0.125, p=q=3$; (c) a $7 \times 3$-grid scroll attractor, where $a=1, b=0.125, p_{1}=q_{1}=3$, $p_{2}=q_{2}=1$.
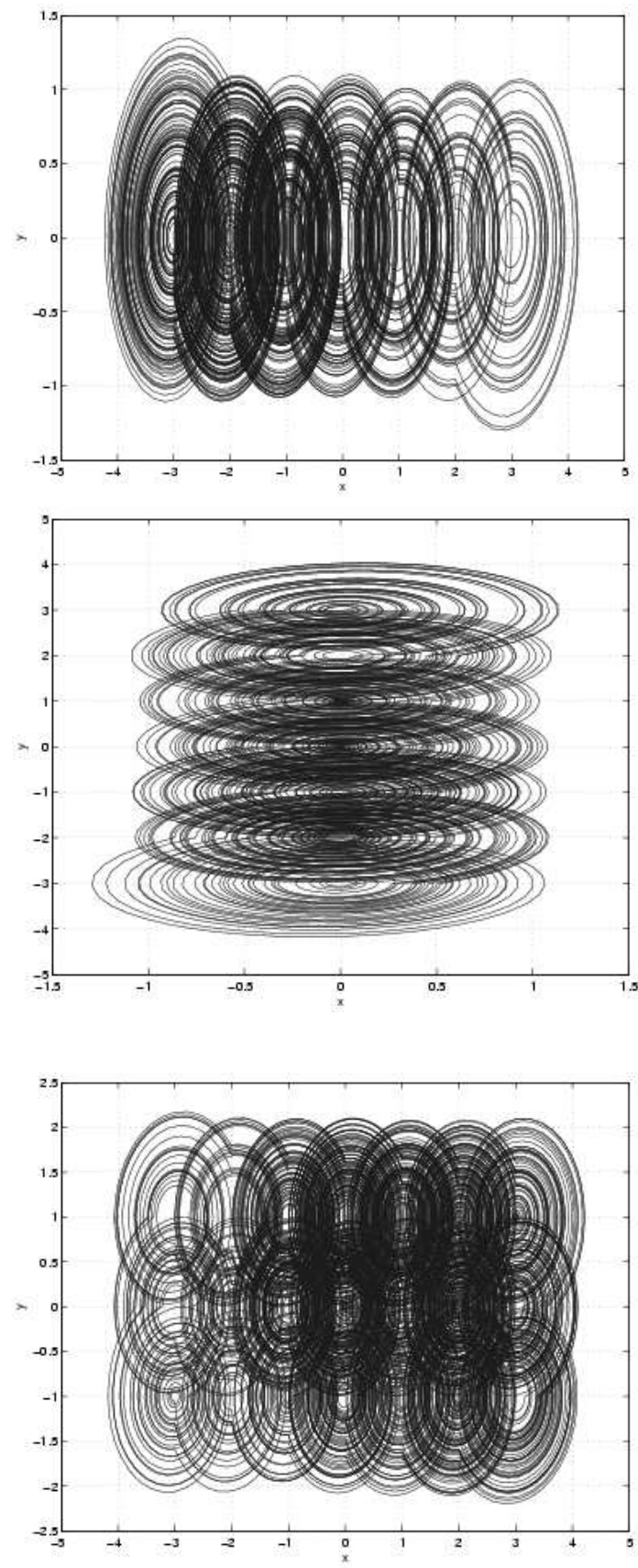

Figure 8. Hysteresis switching chaotic attractors generated from a 2D linear autonomous system. (a) horizontal 7-scroll attractor; (b) vertical 7-scroll attractor; (c) $7 \times 3$-grid scroll attractor.

\section{Remarks 4:}

(1) System (13) is not continuous. Every subspace corresponds to one equilibrium point of the hysteresis system (13), and every equilibrium point corresponds to one scroll of the chaotic attractor of the hysteresis system (13). Moreover, parameters $p_{1}, q_{1}, p_{2}, q_{2}$ determine the numbers of the scrolls, and the hysteresis series $h\left(x, p_{1}, q_{1}\right)$ 
and $h\left(y, p_{2}, q_{2}\right)$ determine the positions of the scrolls. In fact, we can arbitrarily design the number and also the position as well as the orientation of the scrolls of the hysteresis chaotic system (13).

(2) The trajectories of the hysteresis chaotic system are spirally divergent in any subspace; that is, the trajectories are stretched in every subspace. When the trajectories reach the boundaries of a subspace, they will be folded by the switching mechanism of the hysteresis series. Thus, the trajectories will be repeatedly stretched and folded inside a bounded set for infinitely many times, leading to the appearance of bifurcations and chaos.

\subsection{Three-Dimensional Case}

This subsection presents a new systematic method - hysteresis series switching approach - for generating multi-dimensional scroll chaotic attractors from a 3D linear autonomous system [11]. It includes 1D $n$-scroll, 2D $n \times m$-grid scroll, and 3D $n \times m \times l$-grid scroll chaotic attractors.

The 3D linear autonomous system with a hysteresis series switching controller is described by

$$
\dot{X}=A X+B \theta(C X),
$$

where $X=(x, y, z)^{T}$ is the state vector, and

$$
\begin{aligned}
A & =\left(\begin{array}{ccc}
0 & 1 & 0 \\
0 & 0 & 1 \\
-a & -b & -c
\end{array}\right), \quad B=\left(\begin{array}{ccc}
0 & -1 & 0 \\
0 & 0 & -1 \\
a & b & c
\end{array}\right) \\
C & =\left(\begin{array}{ccc}
1 & 0 & 0 \\
0 & 1 & 0 \\
0 & 0 & 1
\end{array}\right) .
\end{aligned}
$$

It can be classified into three different cases as follows:

- 1D $n$-scroll hysteresis chaotic attractor:

$$
\theta(\cdot)=\left(\begin{array}{c}
h\left(x, p_{1}, q_{1}\right) \\
0 \\
0
\end{array}\right),
$$

where $h\left(x, p_{1}, q_{1}\right)$ is similarly defined by (14), whose equilibria are located in the $x$-axis.

- 2D $n \times m$-grid scroll hysteresis chaotic attractor:

$$
\theta(\cdot)=\left(\begin{array}{c}
h\left(x, p_{1}, q_{1}\right) \\
h\left(y, p_{2}, q_{2}\right) \\
0
\end{array}\right),
$$

where $h\left(x, p_{1}, q_{1}\right), h\left(x, p_{2}, q_{2}\right)$ are similarly defined by (14), whose equilibria are integer points located in the $x-y$ plane.
- 3D $n \times m \times l$-grid scroll hysteresis chaotic attractor:

$$
\theta(\cdot)=\left(\begin{array}{c}
h\left(x, p_{1}, q_{1}\right) \\
h\left(y, p_{2}, q_{2}\right) \\
h\left(z, p_{3}, q_{3}\right)
\end{array}\right)
$$

where $h\left(x, p_{1}, q_{1}\right), h\left(x, p_{2}, q_{2}\right), h\left(x, p_{3}, q_{3}\right)$ are similarly defined by (14), whose equilibria are integer points in state space.

Figure 9 displays the hysteresis switching multi-scroll chaotic attractors generated by the controlled system (15): (a) a 1D 7-scroll chaotic attractor, where $a=$ $0.8, b=0.72, c=0.6, p=q=3$; (b) a $2 \mathrm{D}$ $5 \times 7$-grid scroll chaotic attractor, where $a=0.8$, $b=0.7, c=0.6, p_{1}=q_{1}=2, p_{2}=q_{2}=3$; (c), (d) a 3D $5 \times 8 \times 3$-grid scroll chaotic attractor, where $a=0.8, b=0.72, c=0.66, p_{1}=q_{1}=2$, $p_{2}=3, q_{2}=4, p_{3}=q_{3}=1$.
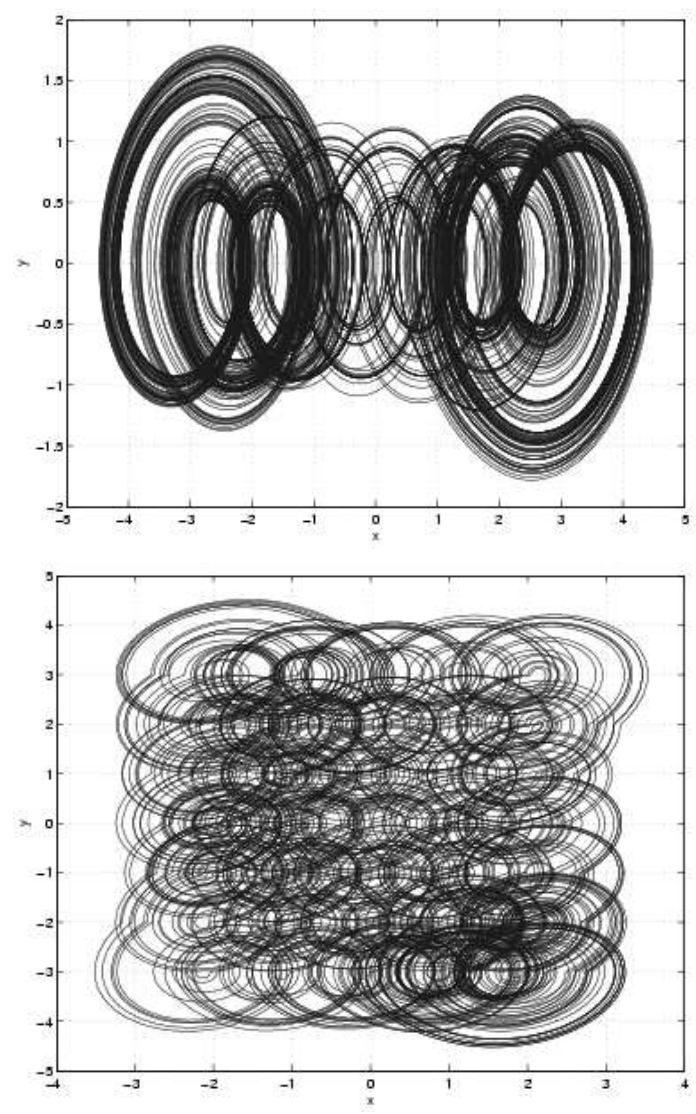

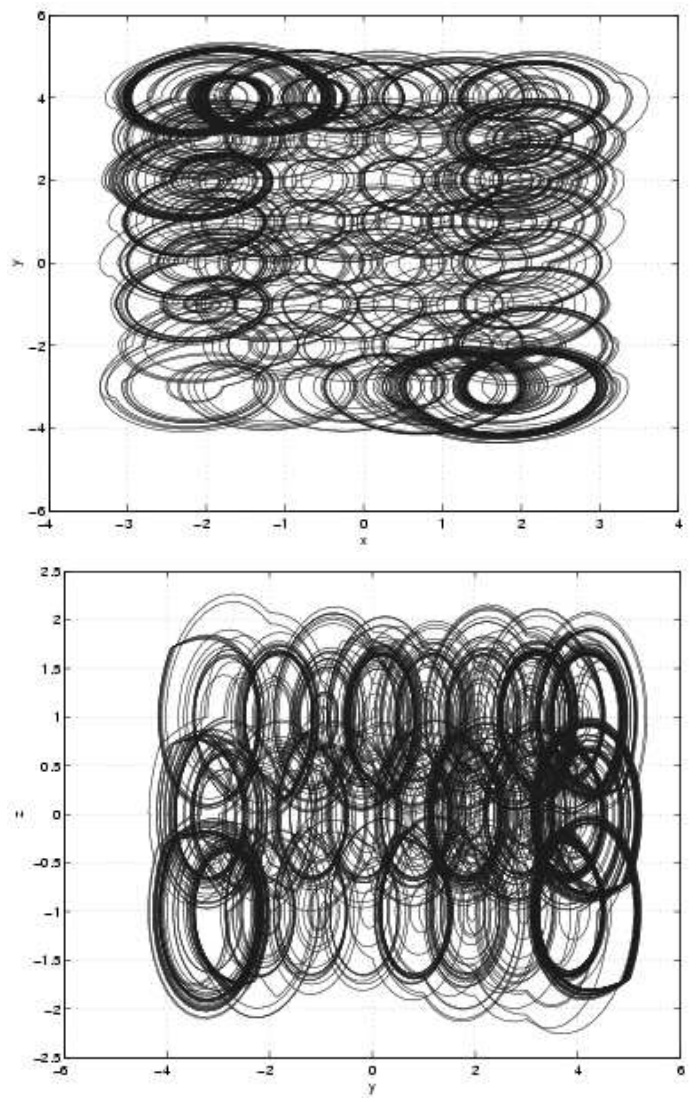

Figure 9. Hysteresis switching chaotic attractors generated from a 3D linear autonomous system. (a)

1D 7-scroll attractor; (b) 2D $5 \times 7$-grid scroll attractor; (c) $x-y$ projection for $3 \mathrm{D}$

$5 \times 8 \times 3$-grid scroll attractor; (d) $y-z$ projection for 3D $5 \times 8 \times 3$-grid scroll attractor.

\section{Remarks 5:}

(1) Parameters $p_{i}, q_{i}(1 \leq i \leq 3)$ of system (15) determine the numbers of the scrolls in $x, y, z$-directions. Moreover, the hysteresis series $h\left(x, p_{1}, q_{1}\right), h\left(y, p_{2}, q_{2}\right)$, and $h\left(z, p_{3}, q_{3}\right)$ determine the positions of the scrolls. In fact, one can arbitrarily design the number and also the position as well as the orientation of the scrolls of the hysteresis switching chaotic system (15).

(2) Every subspace has one and only one equilibrium point, and every equilibrium point corresponds to one scroll of the chaotic attractor of the hysteresis switching system (15). Obviously, the dynamical behaviors of system (15) are rather simple and it has an exact analytic solution in every subspace. However, the dynamical behaviors of assembled system (15) with hysteresis switching are complex.

\section{Conclusions}

This paper has reviewed and also introduced several new approaches for generating multi-scroll chaotic attractors from some simple 2D or 3D linear autonomous systems via switching control. These chaotic systems are rather simple in terms of their algebraic structures - they can be regarded as a linear autonomous system with simple a piecewise linear or piecewise constant switching controller. In fact, all controlled systems discussed in this paper are piecewise linear, and these controlled systems are continuous or discontinuous. However, they can generate fairly complex multi-scroll chaotic attractors.

It has been verified that abundant complex dynamical behaviors can be generated by piecewise-linear functions if designed appropriately. There is great potential to design some simple switching controlled systems, particularly piecewise-linear systems, for generating complex multi-scroll chaotic attractors toward engineering applications.

\section{Acknowledgements}

This work was supported by an Australian International Postgraduate Research Award, the Hong Kong Research Grants Council under the CERG grant CityU 1004/02E, the K. C. Wong Education Foundation of Hong Kong, and the Chinese Postdoctoral Scientific Foundation.

\section{References}

[1] L. O. Chua, M. Komuro, and T. Matsumoto, "The double scroll family," IEEE Trans. Circuits Syst. I, vol. 33, no. 11, pp. 1072-1118, 1986.

[2] J. A. K. Suykens, A. Huang, and L. O. Chua, "A family of $n$-scroll attractors from a generalized Chua's circuit," Archiv fur Elektronik und Ubertragungstechnik, vol. 51, no. 3, pp. 131-138, 1997.

[3] J. A. K. Suykens and J. Vandewalle, "Generation of $n$-double scrolls $(n=1,2,3,4, \ldots)$," IEEE Trans. Circuits Syst. I, vol. 40, no. 11, pp. 861-867, Nov. 1993.

[4] M. A. Aziz-Alaoui, "Multispiral chaos," Proc. 2nd Int. Conf. Control of Oscillations and Chaos, vol. 1, pp. 88-91, 2000.

[5] G. Q. Zhong, K. F. Man, and G. Chen, "A systematic approach to generating $n$-scroll attractors," Int. J. Bifurcation Chaos, vol. 12, no. 12, pp. 1907-2915, Dec. 2002.

[6] M.. E. Yalçin, J. A. K. Suykens, J. Vandewalle, and S. Özoğuz, "Families of scroll grid attractors," Int. J. Bifurcation Chaos, vol. 12, no. 1, pp. 23-41, Jan. 2002. 
[7] J. Lü, T. Zhou, G. Chen, and X. Yang, "Generating chaos with a switching piecewise-linear controller," Chaos, vol. 12, no. 2, pp. 344-349, June 2002.

[8] J. Lü, X. Yu, and G. Chen, "Generating chaotic attractors with multiple merged basins of attraction: A switching piecewise-linear control approach," IEEE Trans. Circuits Syst. I, vol. 50, no. 2, pp. 198-207, Feb. 2003.

[9] T. Saito, "An approach toward higher dimensional hysteresis chaos generators," IEEE Trans. Circuits Syst. I, vol. 37, no. 3, pp. 399-409, Jan. 1990.

[10] F. Han, J. Lü, X. Yu, G. Chen, and Y. Feng, "A new systematic method for generating multi-scroll chaotic attractors from a linear second-order system with hysteresis," preprint, 2003.

[11] J. Lü, F. Han, X. Yu, and G. Chen, "Generating 3-D multi-scroll chaotic attractors: A hysteresis series switching method," preprint, 2003.

[12] K. S. Tang, G. Q. Zhong, G. Chen, and K. F. Man, "Generation of $n$-scroll attractors via sine function," IEEE Trans. Circuits Syst. I, vol. 48, pp. 1369-1372, Nov. 2001. 